\title{
LOW ACTIVE-SENSITIVITY ALLPOLE ACTIVE-RC FILTERS USING IMPEDANCE TAPERING
}

\author{
Dražen Jurišićl ${ }^{1}$ George S. Moschytz ${ }^{2}$ and Neven Mijat ${ }^{1}$ \\ ${ }^{1}$ University of Zagreb, Faculty of Electrical Engineering and Computing, Unska 3, 10000 Zagreb, Croatia \\ ${ }^{2}$ Swiss Federal Institute of Technology Zürich (ETH), Sternwartstrasse 7, 8092 Zürich, Switzerland
}

\begin{abstract}
To reduce the low-power allpole active- $R C$ filter's output magnitude sensitivity to passive components, the "impedance tapering" design method was used. This design procedure has been applied to the design of $2^{\text {nd }}$ - and $3^{\text {rd }}$-order low-pass (LP) (class 4) Sallen-and-Key filters and has already been published [1]. In this paper the active sensitivity analysis using MATHEMATICA was performed on the same "impedance tapered" filter sections as in [1]. The analysis was performed by calculation of the real filter's transfer-function magnitude which was denormalized to various cut-off frequencies from low to high, using a single-pole model of a real operational amplifier. The amount of active and passive sensitivity reduction was compared using the same degree of impedance tapering. It was demonstrated that both sensitivities, i.e. active and passive, were correspondingly reduced for the same filter. Thus, a judicious selection of component values accounts for a considerable decrease in both passive and active sensitivities.
\end{abstract}

\section{INTRODUCTION}

It has already been published in [1] that the allpole active- $R C$ filters of $2^{\text {nd }}$ - and $3^{\text {rd }}$-order can be designed, using the "impedance tapering" technique, for minimum sensitivity to passive component tolerances when compared with standard designs. In this work and in [1], Sallen-and-Key (S\&K) allpole filters are considered, which have a passive $R C$ network in the positive feedback loop (class-4) [2]. As an active element the filters use an operational amplifier (opamp), in order to obtain complex-conjugate poles, high gain and low output resistance. The passive sensitivity was examined using Schoeffler's sensitivity measure with an ideal opamp.

Obviously, in practice the opamp is not ideal. It therefore influences the overall magnitude response of the filter's transfer function. In what follows it will be demonstrated that the influence this non-ideality is very large at high frequencies, and with filters with high pole $\mathrm{Q}$ factors, it is even larger. The question, that now arises is, how the "impedance tapering" design technique, which substantially reduces passive sensitivity, influences the sensitivity to active component variations? Does this special design technique improve the sensitivities to active components as well? To find an answer, we shall use various impedance tapering techniques to design filter circuits and investigate the real opamp influence on their transfer functions. It will be shown that the use of "impedance tapering" in active$R C$ filter design minimizes passive as well as active sensitivities.

\section{SENSITIVITY TO ACTIVE COMPONENT}

\section{$2.12^{\text {nd }}$-order LP Filter Example}

Consider the $2^{\text {nd }}$-order class-4 Sallen \& Key LP filter in Figure 1. The transfer function is given by:

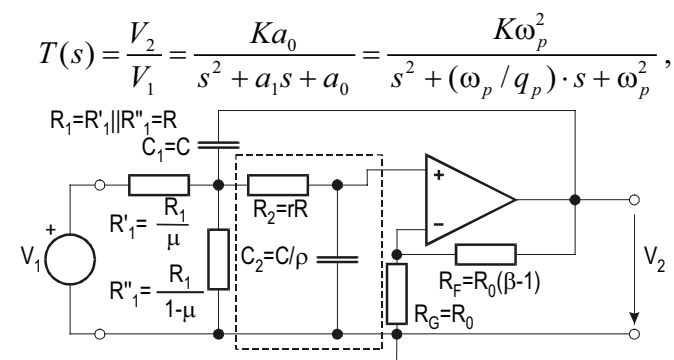

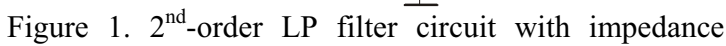
scaling factors ( $r$ for resistors and $\rho$ for capacitors).

where the gain $K$ is given by $K=\mu \beta$. $\beta=1+R_{F} / R_{G}$ represents the positive-feedback gain factor. In what follows consider two practical examples of $2^{\text {nd }}$-order approximations with coefficients: $K=1, f_{p}=1$ ( $i$ ) Butterworth $q_{p}=0.707$; and $(i i) q_{p}=5$. The unity-gain normalized transfer function magnitudes (with the cut-off frequencies $f_{d}=1 \mathrm{~Hz}$ ) are shown in Figure 2(a) and (b), respectively.

Using the step-by-step design procedure given in [1], we calculate the example $(i)$ and obtain filter circuit element values: $R_{1}=R_{2}=0.159, R_{1}^{\prime}=0.252, R^{\prime \prime}{ }_{1}=0.431, C_{1}=C_{2}=1, R_{G}=1, R_{F}=0.586$, $\mu=0.631, \beta=1.586, K=\mu \beta=1$. Note that the filter circuit has equal capacitors and equal resistor values. This is denoted by $\rho=r=1$.
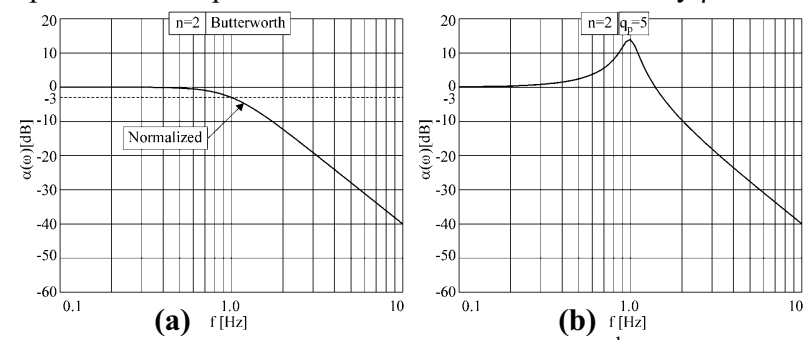

Figure 2. Transfer function magnitude of $2^{\text {nd }}$-order LP filter circuit as in Figure 1. (a) Butterworth. (b) High-Q, $q_{p}=5$.

As a second step in our analysis procedure, we perform a set of de-normalizations on the $2^{\text {nd }}$-order Butterworth LP filter above and obtain a family of filter characteristics, which are presented in Figure 4(a). The obtained characteristics have their cut-off frequencies in the range of $10^{3}$ to $10^{6}[\mathrm{~Hz}$ (i.e. three decades). In every decade we produced 10 logarithmically shifted characteristics (with $f_{d}=\{1.0,1.26,1.59,1.9,2.51,3.16,3.98$, $\left.5.01,6.31,7.94,10.0\} \times 10^{n}[\mathrm{~Hz}] ; n=3,4,5\right)$ and consequently we have a total of 31 characteristics.

Note from Figure 4(a) that all characteristics have the same shape. This is because of the ideal opamp in the filter circuit in Figure 1. In a final step we normalize all curves in Figure 4(a) to the frequency $1 \mathrm{~Hz}$, and obtain all the curves grouped into a single curve, see Figure 2(a). Note that an ideal opamp has infinite open-loop gain $A$, and gain-bandwidth (GB) product, while a real opamp has finite $A$ and GB, and finite input and output impedances. To investigate the influence of impedance 
tapering to active sensitivity in the first approximation of a nonideal amplifier, we introduced a single pole model of a real opamp as a voltage-controlled-voltage-source (VCVS), as presented in Figure 3 into the filter circuit in Figure 1. This circuit represents both an average pure CMOS-based opamp and a high-performance discrete opamp.

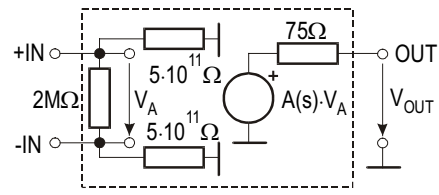

Figure 3. Single-pole model of real opamp.

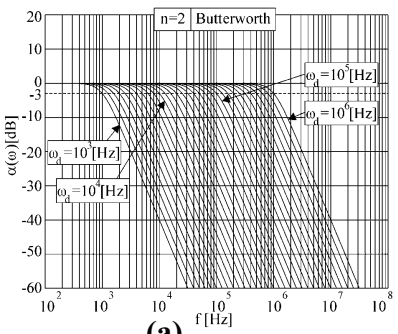

(a)

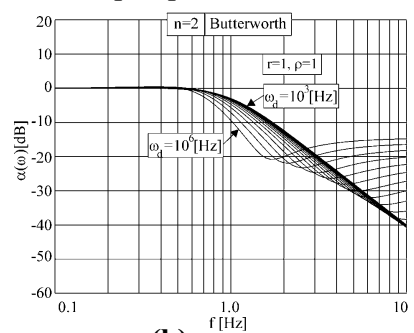

(b)

Figure 4. Family of: (a) denormalized magnitudes with ideal opamp (b) the magnitudes normalized to $1 \mathrm{~Hz}$ with real opamp.

The VCVS in the model in Figure 3 is defined by:

$$
A(s)=V_{\text {OUT }} / V_{A}=A_{0} \omega_{g} \cdot\left(s+\omega_{g}\right)^{-1} ; \quad V_{A}=V_{+I N}-V_{-I N},
$$

where $A_{0}=2.025 \cdot 10^{5}, \omega_{g}=50[\mathrm{rad} / \mathrm{s}]$. If we normalize the curves obtained with a real opamp to the frequency $1 \mathrm{~Hz}$ we obtain the family of frequency responses shown in Figure 4(b).

Observing Figure 4(b) we conclude that at least 9 characteristics of 31 of the filters designed for high frequencies $\left(f_{d}>159 \mathrm{kHz}\right)$ have deteriorated substantially from their nominal shape. The cut-off frequency has shifted to lower frequencies, the gain decreases, and the attenuation in the stop-band actually flattens out. The influence of the "real opamp" model increases even more if the denormalization frequency $f_{d}$ becomes larger, i.e. the overall filter's magnitude becomes significantly deteriorated, especially at higher frequencies. Note that in the simulation of the magnitude response, all passive components take on their nominal values, and that there were no variations in real opamp characteristics. The above assumptions concentrate only on the
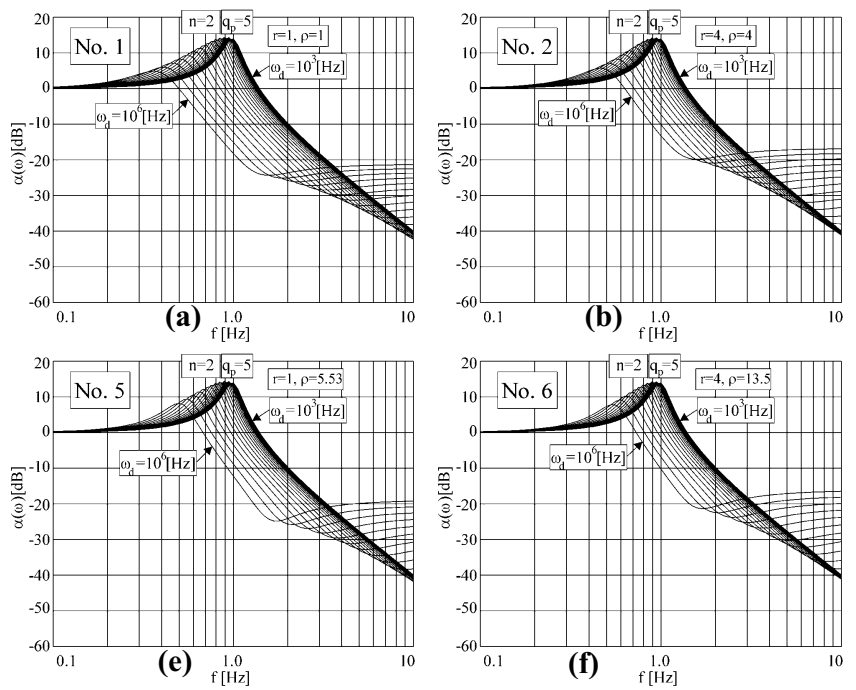

investigation of the finite GB-product and on the filter's magnitude at higher frequencies, at which the open-loop gain $A$ is very low and decreases with increasing frequency. Thus, the open-loop gain variation $A$ is already inbuilt in our investigation, and can assume that the effects of gain $A$ variations will be reduced by impedance tapering, in the same way that non-ideal opamp effects due to finite GB-product, are reduced.

Consider now an example with a relatively high pole $\mathrm{Q}$ value, $q_{p}=5$. The family of denormalized-normalized magnitudes are shown in Figure 5. In Figure 5(a) at least 16 characteristics of 31, i.e. the filters designed for $f_{d}>31.6 \mathrm{kHz}$ have deteriorated. Comparing Figures 4(b) and 5(a) we conclude that higher pole-Q values of the circuit make them more sensitive to component and gain variations under the same design conditions $(\rho=r=1)$.

\subsection{The Gain Sensitivity Product (GSP)}

It is well known that in order to minimize the sensitivity of filter characteristics to tolerances of the gain elements, the gainsensitivity-product (GSP) of the filter should be minimized [3], [4], [5]. In the LP case it is defined by:

$$
G S P=\Gamma_{A}^{q_{p}}=A S_{A}^{q_{p}}=q_{p} \beta^{2} \sqrt{\rho / r} .
$$

In [3], well-proven biquadratic filter circuits ("biquads") and the corresponding design flow-chart listings are given. We use the so-called low-pass medium-Q (LP-MQ) circuit, which essentially corresponds to the circuit in Figure 1 (see [3] pp. 52).

In the design flow-charts, the specification in terms of pole and zero frequencies and Q's is admitted as input, and the circuit with the minimum GSP results as output. In most of the circuits, at least one additional degree of freedom is available, namely the value and ratio of two (or three) capacitors. In the present context this permits the implementation of capacitive impedance tapering while, at the same time, minimizing the GSP. To design a min. GSP LP circuit we choose a value of $\rho$ and then calculate $r$, or alternatively, we choose $r$ and then calculate the $\rho$, using (see [3]):

$$
r=\frac{\rho}{36 q_{p}^{2}}\left[\sqrt{1+12 q_{p}^{2}\left(1+\frac{1}{\rho}\right)}+1\right]^{2} ; \rho=\frac{r}{4 q_{p}^{2}}\left[\sqrt{1+12 q_{p}^{2}\left(1+\frac{1}{r}\right)}-1\right]^{2} .
$$
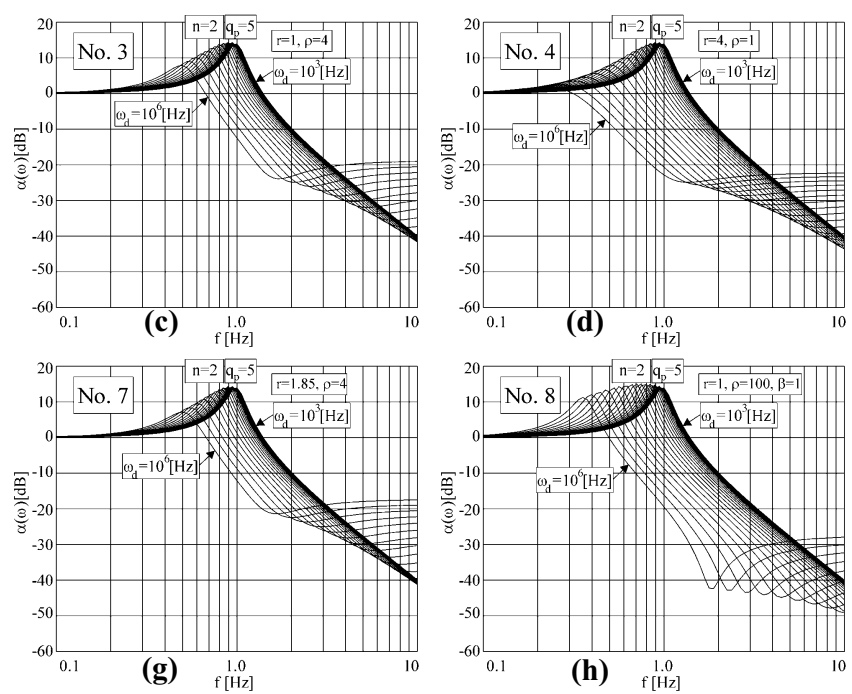

Figure 5. (a)-(h) Family of normalized magnitudes of $2^{\text {nd }}$-order LP filters in Table 1 with real opamp, and $q_{p}=5$. 
In the following example we present different GSP curves with tapering factor $\rho$ varying from 1 to 10 in Figure 6(a) and with tapering factor $r$ varying from 1 to 4 in Figure 6(b). The Pole Q is $q_{p}=5$. Note that every GSP curve in Figure 6 has a corresponding min. GSP value, which follows from eq. (3).

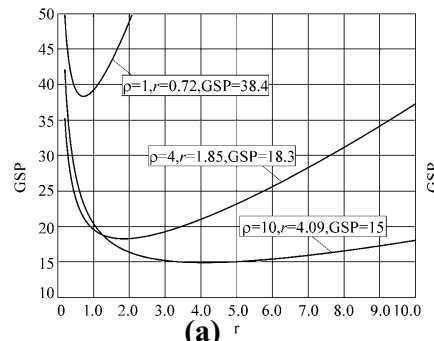

(a)

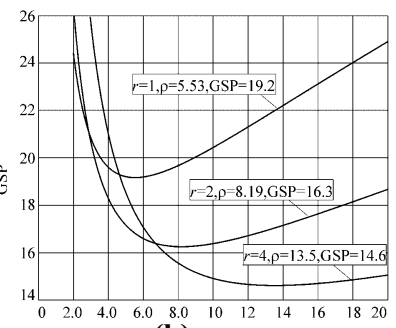

(b) $P$
Figure 6. Plot of gain-sensitivity-product (GSP) for pole Q-factor, $q_{p}=5$. (a) GSP vs. $r$. (b) GSP vs. $\rho$.

The same $2^{\text {nd }}$-and $3^{\text {rd }}$-order low-pass filter examples as used in [1] for the passive sensitivity analysis, are repeated here for the active sensitivity analysis. The $2^{\text {nd }}$-order filter component values are presented in Table 1 . The $\sigma_{\alpha}\left(\omega_{p}\right)[\mathrm{dB}]$ column in Table 1 represents the standard deviation of the variation of the logarithmic gain $\Delta \alpha=8.68588 \Delta|T(j \omega)| / T(j \omega) \mid[\mathrm{dB}]$, with respect to zero mean and $1 \%$ standard deviation of the passive components, at pole frequency $\omega_{p}$. To examine active sensitivities for the filters in Table 1 families of denormalizednormalized magnitudes are shown in Figure 5.

Table 1 Component values of $2^{\text {nd }}$-order LP filters (resistors in $[\Omega]$, capacitors in $[\mathrm{F}], \sigma_{\alpha}\left(\omega_{p}\right)$ in $[\mathrm{dB}]$ ).

\begin{tabular}{|c||c|c|c||c|c|c|c|c|c|}
\hline No. & Filter & $r$ & $\rho$ & $R_{1}$ & $C_{1}$ & $\beta$ & $\mu$ & $\mathrm{GSP}$ & $\sigma_{\alpha}\left(\omega_{p}\right)$ \\
\hline \hline 1. & Non Tapered & 1 & 1 & 0.159 & 1 & 2.80 & 0.36 & 39.2 & 1.754 \\
\hline 2. & Impedance Tapered & 4 & 4 & 0.169 & 1 & 2.05 & 0.49 & 21.0 & 1.146 \\
\hline 3. & Part. Tapered $(r=1)$ & 1 & 4 & 0.318 & 1 & 1.40 & 0.71 & 19.6 & 0.807 \\
\hline 4. & Part. Tapered $(\rho=1)$ & 4 & 1 & 0.079 & 1 & 5.60 & 0.18 & 78.4 & 2.415 \\
\hline 5. & $r=1$ and min. GSP & 1 & 5.53 & 0.374 & 1 & 1.28 & 0.78 & 19.2 & 0.665 \\
\hline 6. & $R$-Taper, min. GSP & 4 & 13.5 & 0.292 & 1 & 1.26 & 0.79 & 14.6 & 0.558 \\
\hline 7. & $C$-Taper, min. GSP & 1.85 & 4 & 0.234 & 1 & 1.58 & 0.63 & 18.3 & 0.891 \\
\hline 8. & Unity gain $(\beta=1)$ & 1 & 100 & 1.590 & 1 & 1.00 & 1.00 & 50.0 & 0.106 \\
\hline
\end{tabular}

Considering the standard deviation $\sigma_{\alpha}\left(\omega_{p}\right)$ of the variation of the logarithmic gain $\alpha[\mathrm{dB}]$, given in Table 1 , we conclude that the ideally impedance-tapered filter (no. 2) has considerably decreased passive sensitivities, compared to the non-tapered standard circuit version (no. 1). Observing Figure 5 the same conclusions can be derived for active sensitivities. By tapering only the capacitors, while keeping the resistor values equal $(r=1)$, (no. 3), both passive and active filter sensitivities are decreased even more. The resistively tapered filter (no. 4) has the highest sensitivities, for the reason given in [1]. This tracking between passive and active sensitivities is significant for the design of active filters with overall low sensitivity to active and passive component variations.

Note that, the equal-resistor and min. GSP filter (no. 5) and the R-tapered and min. GSP filter (no. 6) show somewhat lower passive sensitivity, and very reduced active sensitivity (especially filter no. 6). When calculating the GSP-minimum circuits using [3], we have chosen the values and ratio $\rho=4$ of two capacitors and obtained circuit (no. 7), which has even smaller passive and active sensitivities. The best passive sensitivity is obtained for the $\beta=1$ filter (no. 8) but here the active sensitivity has deteriorated considerably.
In summary, for the general $2^{\text {nd }}$-order allpole low-pass filter, the minimum of both passive and active sensitivities is obtained with capacitive impedance tapering and resistor values selected for GSP-minimization.

\section{$2.33^{\text {rd }}$-order LP Filter Example}

Consider the $3^{\text {rd }}$-order class- 4 Sallen \& Key LP filter in Figure 7.

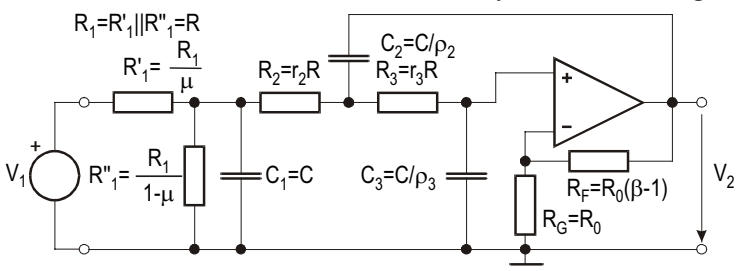

Figure 7. $3^{\text {rd }}$-order LP filter circuit with impedance scaling factors.

The transfer function is given by:

$$
T(s)=\frac{V_{2}}{V_{1}}=\frac{K a_{0}}{s^{3}+a_{2} s^{2}+a_{1} s+a_{0}}=\frac{K \gamma \omega_{p}^{2}}{(s+\gamma)\left[s^{2}+\left(\omega_{p} / q_{p}\right) \cdot s+\omega_{p}^{2}\right]} .
$$

Consider the $3^{\text {rd }}$-order Chebyshev approximations with passband ripple $0.5 \mathrm{~dB}$ as in [1], which has the parameters: $q_{p}=1.70619 ; \omega_{p}=1.06885 ; \gamma=0.62646$.

Table 2 Component values of $3^{\text {rd }}$-order LP filters

(resistors in [ $\Omega]$, capacitors in $[\mathrm{F}], \sigma_{\alpha}\left(\omega_{p}\right)$ in $[\mathrm{dB}]$ ).

\begin{tabular}{|c||c||c|c|c|c|c|c|c|c|c|}
\hline No. & Filter & $R_{1}$ & $C_{1}$ & $\rho_{2}$ & $\rho_{3}$ & $r_{2}$ & $r_{3}$ & $\beta$ & $\mu$ & $\sigma_{\alpha}\left(\omega_{p}\right)$ \\
\hline \hline 1. & $C$-equal & 0.298 & 1 & 1 & 1 & 1.48 & 0.14 & 2.0 & 0.5 & 1.220 \\
\hline 2. & $C$-taper: $\rho_{C}=3$ & 0.391 & 1 & 3 & 9 & 0.93 & 2.72 & 2.0 & 0.5 & 0.528 \\
\hline 3. & $C$-taper: $\rho_{C}=5$ & 0.344 & 1 & 5 & 25 & 1.66 & 10.4 & 2.0 & 0.5 & 0.465 \\
\hline 4. & $R$-equal & 0.413 & 1 & 4.64 & 2.68 & 1 & 1 & 4.0 & 0.25 & 1.044 \\
\hline 5. & $R$-taper: $\rho_{C}=3$ & 0.328 & 1 & 12.8 & 13.3 & 3 & 9 & 4.0 & 0.25 & 0.783 \\
\hline 6. & $R$-taper: $\rho_{C}=5$ & 0.303 & 1 & 20.3 & 30.6 & 5 & 25 & 4.0 & 0.25 & 0.734 \\
\hline
\end{tabular}
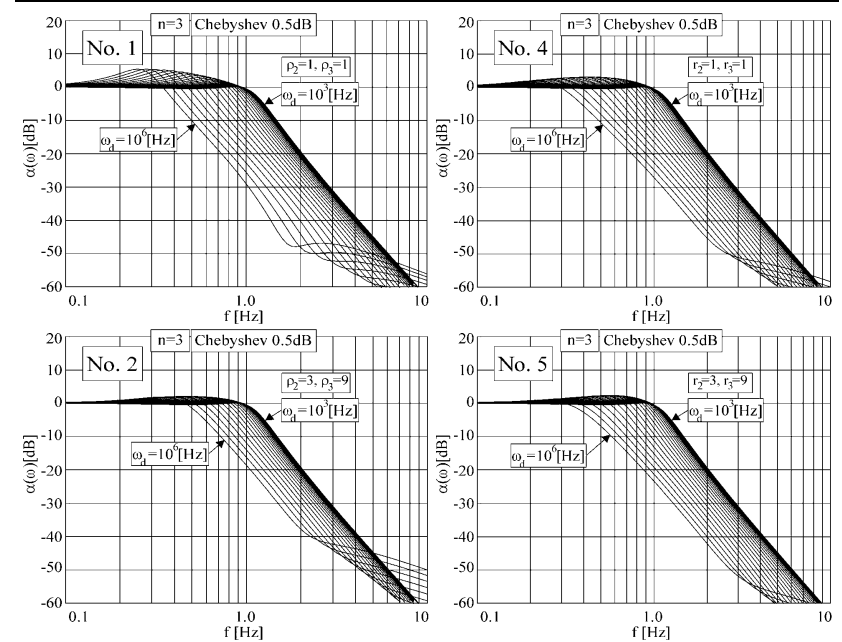

1.0
$\mathrm{f}[\mathrm{Hz}]$
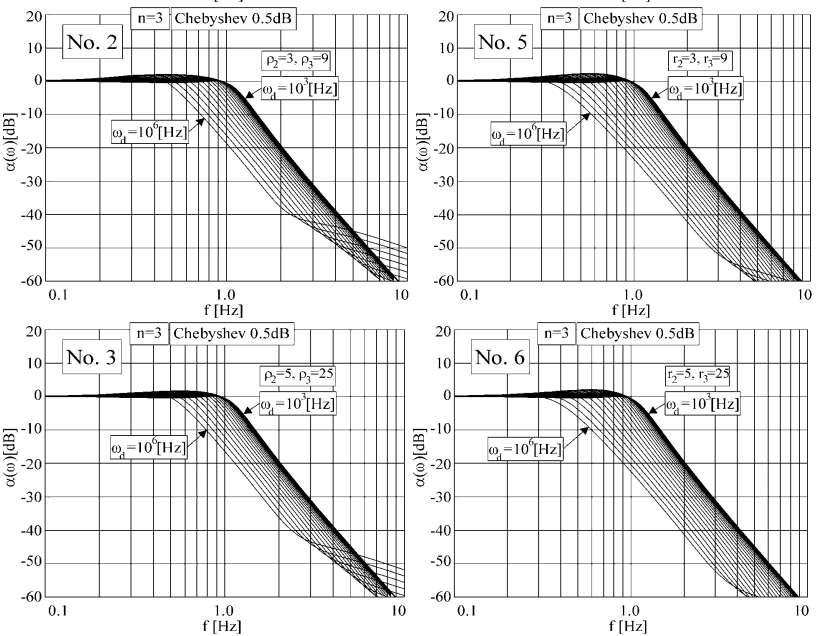

Figure 8. Family of normalized magnitudes of $3^{\text {rd }}$-order LP filters in Table 2 with real opamp. 


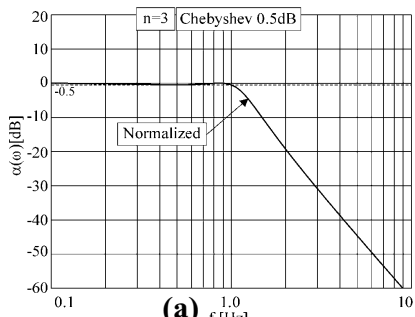

(a) $\begin{gathered}1.0 \\ \mathrm{f}[\mathrm{Hz}]\end{gathered}$

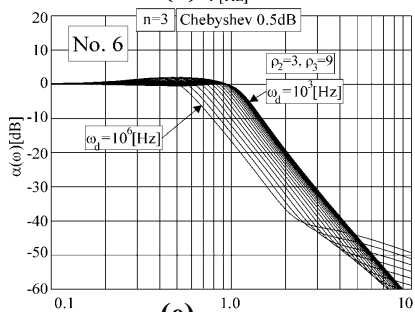

(e) $\begin{gathered}1.0 \\ {[\mathrm{~Hz}]}\end{gathered}$

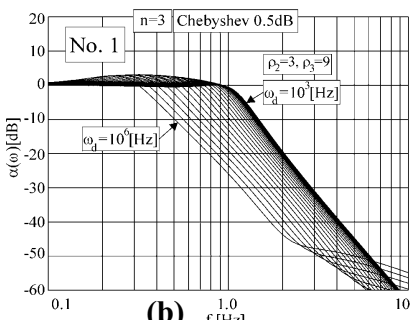

(b) $\begin{gathered}1.0 \\ f[\mathrm{~Hz}]\end{gathered}$

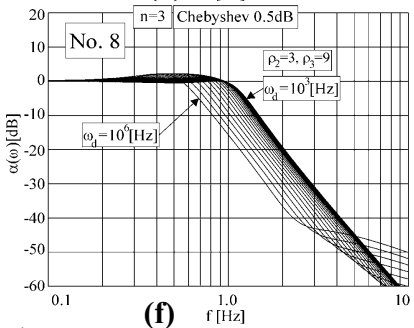

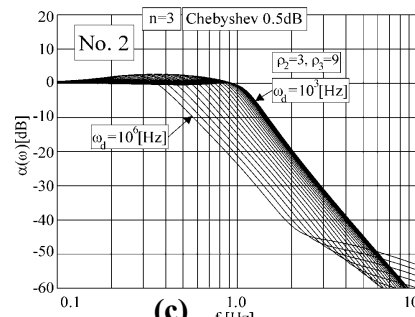

(c) $\begin{gathered}1.0 \\ \text { f }[\mathrm{Hz}]\end{gathered}$

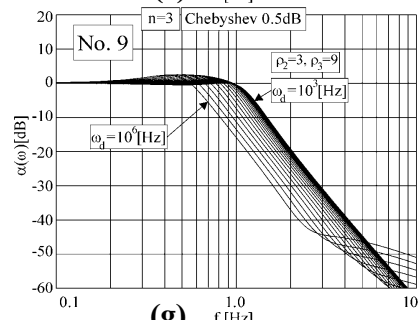

(g) $\begin{array}{r}1.0 \\ \mathrm{f}[\mathrm{Hz}]\end{array}$

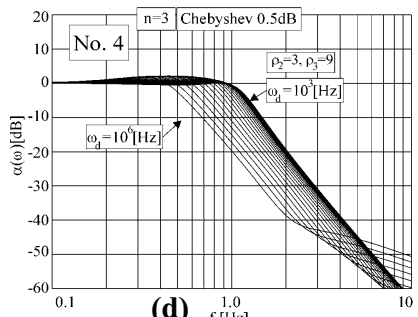

(d) $\begin{aligned} & 1.0 \\ & \mathrm{f}[\mathrm{Hz}]\end{aligned}$

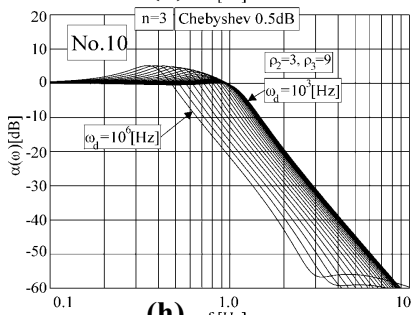

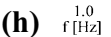

Figure 9. Chebyshev $0.5 \mathrm{~dB} 3^{\text {rd }}$-order LP filter circuit as in Figure 7. (a) Transfer function magnitude. (b)-(h) Family of normalized magnitudes of filters in Table 3 with real opamp.

The transfer function magnitude is shown in Figure 9(a). Capacitive and resistive impedance tapering techniques as in [1] are applied, and the obtained filter components are given in Table 2.

The corresponding active sensitivity analysis is presented in Figure 8. Observing the families of curves in Figure 8, we conclude that the impedance tapering has minimized the active sensitivity in the same way as it minimized the passive sensitivity. Active desensitization is compared with the results of passive desensitization in [1], which is presented in the form of $\sigma_{\alpha}\left(\omega_{p}\right)$ columns in Table 1-Table 3.

Finally, we constructed filter circuits applying capacitive tapering and using the 10 different design frequencies $\omega_{0}=\left(R_{1} C_{1}\right)^{-1}$ as in [1]. We present 7 of 10 obtained filter circuits in Table 3. Note that we use the circuit numbers as in [1]. Note that $f_{0}=\omega_{0} /(2 \pi)$.

Table 3 Component values of $3^{\text {rd }}$-order LP filters $\left(f_{0}\right.$ in $[\mathrm{Hz}], \sigma_{\alpha}\left(\omega_{p}\right)$ in $\left.[\mathrm{dB}]\right)$.

\begin{tabular}{|c|c|c|c|c|c|c|c|c|c|}
\hline No. & Filter & $f_{0}$ & $\rho_{2}$ & $\rho_{3}$ & $r_{2}$ & $r_{3}$ & $r_{3} / r_{2}$ & $\beta$ & $\sigma_{\alpha}\left(\omega_{p}\right)$ \\
\hline 1. & \multirow{7}{*}{$C$-taper: $\rho_{C}=3$} & 1.34 & 3 & 9 & 0.256 & 1.4 & 5.47 & 3.0 & 1.016 \\
\hline 2. & & 1.67 & 3 & 9 & 0.382 & 1.84 & 4.81 & 2.71 & 0.836 \\
\hline 4. & & 2.33 & 3 & 9 & 0.754 & 2.56 & 3.4 & 2.17 & 0.592 \\
\hline 6. & & 3.0 & 3 & 9 & 1.455 & 2.82 & 1.94 & 1.69 & 0.418 \\
\hline 8. & & 3.46 & 3 & 9 & 2.604 & 2.44 & 0.94 & 1.41 & 0.330 \\
\hline 9. & & 3.58 & 3 & 9 & 3.806 & 1.97 & 0.52 & 1.31 & 0.318 \\
\hline 10. & & 3.88 & 3 & 9 & 8.537 & 1.03 & 0.12 & 1.27 & 0.521 \\
\hline
\end{tabular}

The corresponding active sensitivity is shown in the curves of Figure 9(b) to (h). From these curves, we conclude that the deviation from the ideal filter response becomes smallest for the filter no. 8, which is a capacitively tapered filter with resistor scaling values $r_{2} \approx r_{3}$. Considering the correspondence between active and passive desensitization using impedance tapering demonstrated above, it comes as no surprise that here again the filter no. 8 has smallest sensitivity also to passive component tolerances (see [1]).
In summary, for the general $3^{\text {rd }}$-order allpole low-pass filter, the minimum of both passive and active sensitivities is obtained with capacitive impedance tapering and resistor values $r_{2} \approx r_{3}$.

\section{CONCLUSIONS}

A procedure for the design of allpole filters with low sensitivity to passive component tolerances was presented in [1]. Here we have shown that the same design procedure can be used to minimize the influence of gain variation especially at high frequencies. From the results obtained, we can summarize that for the general $2^{\text {nd }}$-order LP filter, the minimum of both passive and active sensitivities is obtained for capacitive impedance tapering with resistor values selected for GSP-minimization. For the general $3^{\text {rd }}$-order LP filter, capacitive impedance tapering with close resistor values $r_{2} \approx r_{3}$ is also effective in passive and active sensitivity minimization. We conclude that impedance tapering is a very effective strategy for the comprehensive sensitivity minimization of a filter circuit, compared with the standard design methods, which are actually given in design handbooks. It is also shown that the active sensitivity is proportional to the pole Q factor. Thus, as is well known, in filter design solutions with lower pole Q-factors, are preferable.

\section{REFERENCES}

[1] G. S. Moschytz, "Low-Sensitivity, Low-Power, Active-RC Allpole Filters Using Impedance Tapering," IEEE Trans. on Circuits and Systems-II: Analog and Digital Signal Processing, vol. CAS-46, no. 8, pp. 1009-1026, Aug. 1999.

[2] R. P. Sallen and E. L. Key, "A practical Method of Designing $R C$ Active Filters," IRE Trans. on Circuit Theory, vol. CT-2, pp. 78-85, 1955.

[3] G. S. Moschytz and P. Horn, Active Filter Design Handbook. Chichester, U.K.: Wiley 1981.

[4] G. S. Moschytz, Linear Integrated Networks: Fundamentals. NY (Bell Labs Series): V. Nostrad Reinhold Co., 1974.

[5] G. S. Moschytz, Linear Integrated Networks: Design. New York (Bell Labs Series): Van Nostrad Reinhold Co., 1975. 